\title{
Evidências de Interação Genótipo x Ambiente sobre Características de Crescimento em Bovinos de Corte ${ }^{1}$
}

\author{
Maurício Mello de Alencar ${ }^{2}$, Arthur dos Santos Mascioli ${ }^{3}$, Alfredo Ribeiro de Freitas ${ }^{4}$
}

RESUMO - Objetivou-se, com este trabalho, estudar a interação genótipo x ambiente sobre os pesos à desmama (PD) e aos 12 meses de idade (P12), o ganho de peso diário da desmama aos 12 meses de idade (GDA) e o desempenho com base em um índice (CPG) de componentes principais envolvendo essas três características, em um rebanho da raça Canchim. O ambiente foi considerado a época de nascimento (primeiro e segundo semestres) do animal. Para avaliar a interação genótipo x ambiente, foram utilizadas duas metodologias: 1 - estimativas de parâmetros genéticos pelo método da máxima verossimilhança restrita livre de derivadas (REML), com análise bicaráter (mesma característica nas duas épocas), com o modelo estatístico incluindo os efeitos fixos de sexo, ano e mês de nascimento do animal e idade da vaca ao parto como covariável (linear e quadrático) e os efeitos aleatórios de animal e do resíduo; e 2 - semelhante à metodologia 1, porém, no modelo estatístico, incluiu-se ou não o efeito aleatório não correlacionado de touro-época de nascimento, testando a diferença entre os dois modelos pelo teste de razão de verossimilhança. Pela Metodologia 1, as correlações genéticas obtidas para a mesma característica nas duas épocas de nascimento foram iguais a 0,$87 ; 0,97 ; 0,91$ e 0,88 , para PD, P12, GDA e CPG, respectivamente. As estimativas de herdabilidade obtidas foram semelhantes para as duas épocas de nascimento, para todas as características estudadas. Pela metodologia 2, o efeito de touro-época de nascimento foi significativo para todas as características estudadas. Estes resultados evidenciam a existência de interação genótipo x época de nascimento para as características estudadas, sugerindo que as avaliações genéticas e a seleção dos animais desse rebanho devem considerar essa interação.

Palavras-chave: bovinos Canchim, correlação genética, época de nascimento, herdabilidade, peso

\section{Evidences of Genotype x Environment Interaction for Growth Traits in Beef Cattle}

ABSTRACT - The objective of this study was to evaluate the genotype $\mathrm{x}$ environment interaction for body weight at weaning (WW) and 12 months of age (W12), average daily gain from weaning to 12 months of age (ADG), and performance based on a principal components index (PC) involving these three traits, in a Canchim (5/8 Charolais $+3 / 8 \mathrm{Zebu})$ herd. The environment was the season (semester) of birth, and two methods were used to evaluate the genotype x environment interaction: Method 1 - genetic parameters estimated by the derivative free maximum likelihood method (REML), using two-trait analyses (the same trait in the two seasons), and a model that included the effects of year and month of birth, sex and age of cow as a covariate (linear and quadratic effects), and the random effect of animal; and Method 2 - same methodology as Method 1, but with two statistical models, with or without the uncorrelated random effect of sire - season of birth, testing the difference between the two models using the likelyhood ratio test. By Method 1, the genetic correlations for the same trait in the two environments (seasons) were equal to 0.87, 0.97, 0.91 and 0.88 for WW, W12, ADG and PC, respectively. The heritability estimates were very similar for both environments, for all traits studied. By Method 2, the sire-season of birth effect was significant for all traits studied. These results show evidence of genotype $\mathrm{x}$ season of birth interaction for the traits studied, suggesting that, in this herd, genetic evaluation and selection should take this interaction into account.

Key Words: body weight, Canchim cattle, genetic correlation, heritability, season of birth

\section{Introdução}

Ambos, genética e ambiente, são importantes na expressão da maioria das características econômicas em bovinos de corte, e uma questão básica no melhoramento genético animal é se a seleção dos animais praticada em determinado ambiente resulta em progresso genético em outro tipo de ambiente. A interação genótipo x ambiente é de especial interesse quando genótipos superiores em determinado ambiente podem não o ser em ambiente diferente. Essa interação pode também provocar alterações nas variações genéticas, fenotípicas e ambientais e, por conseguinte, resultar em mudanças nas estimativas dos parâmetros genéticos e fenotípicos, implicando na possibilidade de mudanças nos critérios de sele-

\footnotetext{
1 Parte da tese de doutorado do segundo autor, UNESP/Jaboticabal. Bolsista da FAPESP

2 Pesquisador da Embrapa Pecuária Sudeste. Bolsista do CNPq. E.mail: mauricio@cppse.embrapa.br

3 Professor de Melhoramento Genético Animal. E.mail: mascioli@netsite.com.br

4 Embrapa Pecuária Sudeste. Bolsista do CNPq. E.mail: ribeiro@cppse.embrapa.br
} 
ção, dependendo do ambiente. Portanto, a identificação dessas interações deve contribuir para o aumento da eficiência de seleção em bovinos.

No Brasil, com o início da utilização de dados de campo na avaliação de touros, alguns pesquisadores (Rosa et al., 1982; Alencar, 1985; Nobre et al., 1987 e 1988; Silva, 1990; Euclides Filho et al., 1991; Eler et al., 2000; Souza et al., 2003) fizeram estudos para avaliar a existência de interação genótipo x ambiente para características de peso em bovinos de corte. Entretanto, apesar dos estudos nessa área, ainda é pouco conhecida a importância dessa interação na eficiência produtiva de bovinos de corte, e em que amplitude de genótipos e de ambientes ela é importante.

Este trabalho foi conduzido com o objetivo de avaliar a importância da interação genótipo x ambiente para características de peso na raça Canchim, para animais nascidos em duas épocas do ano (primeiro e segundo semestres).

\section{Material e Métodos}

Os dados utilizados neste estudo são provenientes do rebanho de bovinos da raça Canchim pertencente à Embrapa Pecuária Sudeste, São Carlos, SP. Os animais foram criados em regime exclusivo de pastagens e por vários anos foram utilizadas duas estações de monta, uma no primeiro semestre e outra no segundo semestre, com início e duração variáveis, ocorrendo nascimentos em todos os meses do ano. $\mathrm{O}$ rebanho é fechado desde sua formação, porém, no período considerado neste trabalho, foram evitados acasalamentos consangüíneos e adotados os cuidados sanitários normais da região. Neste trabalho, foram considerados apenas os dados de desempenho dos animais criados em regime exclusivo de pastagens; dos animais suplementados, consideraram-se apenas as identificações e os "pedigrees" para calcular a matriz de parentesco.

Foram estudados dados de pesos à desmama (PD; padronizado para 240 dias) e aos 12 meses de idade (P12; padronizado para 365 dias) e de ganho de peso diário da desmama aos 12 meses de idade (GDA), de animais nascidos em duas épocas do ano (primeiro e segundo semestres). Utilizando-se esses dados e a metodologia de componentes principais com a matriz de correlação, pelo procedimento PRINCOMP (SAS, 1996), foi obtido o índice CPG = 0,5342PD + 0,7388P12 + 0,4199GDA, que explicou $79,0 \%$ da variação total, indicando maior contribui- ção de P12, seguido de PD e de GDA. Essa característica ou índice também foi incluída na análise. O estudo da interação genótipo $\mathrm{x}$ ambiente foi realizado utilizando-se dois métodos, descritos a seguir; entretanto, inicialmente foram realizadas análises exploratórias dos dados observados de todas as características estudadas, por meio do procedimento UNIVARIATE (SAS, 1996). Foram obtidos coeficientes de assimetria e de curtose para verificar se os dados ajustavam à distribuição normal. Para eliminar possíveis "outliers", optou-se pela eliminação dos dados fora da amplitude de três desvios-padrão.

O método 1 utilizou a metodologia da máxima verossimilhança restrita e modelo animal para obter estimativas dos componentes de (co)variâncias e demais parâmetros genéticos das características estudadas, cujo modelo estatístico incluiu os efeitos fixos de sexo, ano e mês de nascimento do animal, a covariável (efeitos linear e quadrático) idade da vaca ao parto, em dias, e os efeitos aleatórios aditivo direto e residual. Neste caso, a mesma característica nos dois semestres de nascimento foi considerada como sendo características distintas, realizando-se então, análises bicaráter para PD, P12, GDA e CPG, com a covariância residual entre elas igual a zero. Também, para essas características, foram realizadas análises unicaráter dentro de cada época, utilizando-se o mesmo modelo.

Para ambas as análises (uni e bicaráter) de cada característica, os touros que possuíam progênies nas duas épocas de nascimento foram classificados em ordem decrescente de valores genéticos dentro de cada época, sendo, então, calculada a correlação de Spearman entre as classificações dos touros nas duas épocas.

A existência ou não de interação genótipo $\mathrm{x}$ ambiente foi avaliada pela correlação genética da mesma característica nos dois ambientes (época de nascimento) e pelo valor da correlação de Spearman entre os valores genéticos dos touros nos dois ambientes.

A estrutura dos dados considerando-se a mesma característica em cada época de nascimento como características distintas, assim como estatísticas descritivas das características de desempenho, são apresentadas na Tabela 1, para as análises uni e bicaráter.

O método 2 utilizou a mesma metodologia do método 1, mas neste caso, além dos efeitos fixos de sexo, ano e mês de nascimento e da covariável idade

\section{R. Bras. Zootec., v.34, n.2, p.489-495, 2005}


da vaca ao parto (linear e quadrático) e do efeito aleatório de animal, considerados em um modelo (Modelo 1), outro modelo (Modelo 2) incluiu o efeito aleatório não correlacionado de touro - época de nascimento. A interação genótipo $\mathrm{x}$ ambiente foi avaliada por meio do teste da razão de verossimilhança (Mood et al., 1974), em que a função 2(Log Modelo 2 - Log Modelo 1) apresenta distribuição de $\chi^{2}$ com um grau de liberdade, dado pela diferença no número de parâmetros dos modelos. Foi feita também a comparação do Modelo 1 (dois parâmetros) deste método com o modelo bicaráter (cinco parâmetros) do método 1, utilizando-se o teste da razão de verossimilhança com três graus de liberdade para a distribuição de $\chi^{2}$. Para todas essas análises, foram consideradas apenas dados de touros com no mínimo quatro filhos. Portanto, o número de observações é inferior ao apresentado na Tabela 1.

As estimativas dos componentes de (co)variância foram obtidas utilizando-se o programa MTDFREML (Boldman et al., 1993).

\section{Resultados e Discussão}

Na Tabela 2 são apresentados os componentes de (co)variância de PD, P12, GDA e CPG, de acordo com a época de nascimento, obtidos pelo Método 1. Observam-se alterações nas variâncias genéticas e residuais em relação às épocas de nascimento.

Considerando-se a análise bicaráter, para PD, as estimativas das variâncias genética aditiva e residual foram $15,46 \%$ e $18,33 \%$ maiores para o semestre 2 , respectivamente. Para $\mathrm{P} 12$, maiores estimativas das variâncias genética $(13,07 \%)$ e residual $(18,90 \%)$ ocorreram no semestre 1. Para GDA, a variância genética aditiva foi $27,20 \%$ menor no semestre 1 , enquanto que a residual foi apenas $1,80 \%$ maior no semestre 2. Observaram-se também alterações nas variâncias genética aditiva $(5,78 \%)$ e residual $(8,11 \%)$, para o índice $\mathrm{CPG}$, que foram maiores para o semestre 1. Verifica-se que as relações entre as variâncias de PD seguem tendência contrária às de P12; no caso de $\mathrm{CPG}$, essas relações seguem a mesma tendência

Tabela 1 - Estrutura dos dados dos pesos à desmama (PD) e aos 12 meses de idade (P12), do ganho de peso médio diário da desmama aos 12 meses de idade (GDA) e do primeiro componente principal (CPG) combinando $\mathrm{PD}, \mathrm{GDA}$ e P12, para os animais Canchim nascidos no $1^{\circ}$ (S1) e no $2^{\circ}$ (S2) semestres do ano

Table 1 - Structure of the data for weaning (WW) and 12-month (W12) weights, daily gain from weaning to 12 months of age (ADG) and first principal component (PC) combining WW, W12 and ADG, for Canchim cattle born in the $1^{\text {st }}\left(\right.$ (S1) and $2^{\text {nd }}$ (S2) semesters

\begin{tabular}{|c|c|c|c|c|c|c|c|c|}
\hline & \multicolumn{4}{|c|}{ S1 } & \multicolumn{4}{|c|}{ S2 } \\
\hline & PD & $\mathrm{P} 12$ & GDA & $\mathrm{CPG}$ & PD & P12 & GDA & $\mathrm{CPG}$ \\
\hline & $W W$ & W12 & $A D G$ & $P C$ & $W W$ & W12 & $A D G$ & $P C$ \\
\hline Registros (Records) & 1791 & 1639 & 1607 & 1605 & 3788 & 3369 & 3278 & 3278 \\
\hline Ano nasc. (Year of birth) & 35 & 35 & 35 & 35 & 38 & 38 & 38 & 38 \\
\hline Mês nasc. (Month of birth) & 6 & 6 & 6 & 6 & 6 & 6 & 6 & 6 \\
\hline $\operatorname{Sexo}(\operatorname{Sex})$ & 2 & 2 & 2 & 2 & 2 & 2 & 2 & 2 \\
\hline Média, kg (Mean, kg) & 198 & 234 & 0,29 & 278 & 205 & 205 & 0,02 & 259 \\
\hline Mín., kg (Min., kg) & 90 & 104 & $-0,70$ & 129 & 90 & 95 & $-0,75$ & 126 \\
\hline Máx., kg (Max., kg) & 305 & 368 & 1,32 & 431 & 318 & 327 & 1,30 & 400 \\
\hline $\mathrm{DP}^{1}, \mathrm{~kg}\left(S D^{1}, k g\right)$ & 36 & 45 & 0,22 & 49 & 38 & 40 & 0,22 & 45 \\
\hline $\mathrm{CV}, \%(C V, \%)$ & 18 & 19 & 77 & 18 & 18 & 19 & 915 & 17 \\
\hline
\end{tabular}

1 Desvio-padrão.

1 Standard deviation. 
Tabela 2 - Componentes de (co)variância dos pesos à desmama (PD) e aos 12 meses de idade (P12), do ganho de peso médio diário da desmama aos 12 meses de idade (GDA) e do primeiro componente principal (CPG) combinando PD, GDA e P12, para os animais Canchim nascidos no $1^{\circ}$ (S1) e no $2^{\circ}$ (S2) semestres do ano

Table 2 - Components of variance and covariance for weaning (WW) and 12-month (W12) weights, daily gain from weaning to 12 months of age (ADG) and first principal component $(P C)$ combining WW, W12 and ADG, for Canchim cattle born in the $1^{\text {st }}(\mathrm{S} 1)$ and $2^{\text {nd }}(\mathrm{S} 2)$ semesters

\begin{tabular}{|c|c|c|c|c|c|c|c|c|c|}
\hline \multirow{3}{*}{$\begin{array}{l}\text { Caract. } \\
\text { Trait }\end{array}$} & \multicolumn{5}{|c|}{$\begin{array}{l}\text { Análise bicaráter } \\
\text { Two-trait analysis }\end{array}$} & \multicolumn{4}{|c|}{$\begin{array}{l}\text { Análise unicaráter } \\
\text { One-trait analysis }\end{array}$} \\
\hline & \multicolumn{2}{|c|}{$\begin{array}{l}\text { S1 } \\
\text { S1 }\end{array}$} & \multicolumn{2}{|c|}{$\begin{array}{l}\mathrm{S} 2 \\
\mathrm{~S} 2 \\
\end{array}$} & \multirow{2}{*}{$\begin{array}{c}\mathrm{S} 1 \text { e } \mathrm{S} 2 \\
S 1 \text { and } S 2 \\
\sigma_{\mathrm{a} 12}\end{array}$} & \multicolumn{2}{|c|}{$\begin{array}{l}\text { S1 } \\
\text { S1 }\end{array}$} & \multicolumn{2}{|c|}{$\begin{array}{l}\mathrm{S} 2 \\
\mathrm{~S} 2\end{array}$} \\
\hline & $\sigma_{a}^{2}$ & $\sigma_{\mathrm{e}}^{2}$ & $\sigma_{a}^{2}$ & $\sigma_{\mathrm{e}}^{2}$ & & $\sigma_{\mathrm{a}}^{2}$ & $\sigma_{\mathrm{e}}^{2}$ & $\sigma_{a}^{2}$ & $\sigma_{\mathrm{e}}^{2}$ \\
\hline $\mathrm{PD}(W W)$ & 319 & 466 & 368 & 552 & 297 & 278 & 494 & 335 & 571 \\
\hline $\mathrm{P} 12(W 12)$ & 404 & 705 & 357 & 593 & 368 & 329 & 750 & 329 & 590 \\
\hline $\mathrm{GDA}^{*}(A D G)$ & 3 & 29 & 4 & 28 & 3 & 2 & 29 & 3 & 28 \\
\hline $\mathrm{CPG}(P C)$ & 583 & 746 & 551 & 690 & 498 & 482 & 809 & 497 & 723 \\
\hline
\end{tabular}

$\sigma_{\mathrm{a}}{ }^{2}, \sigma_{\mathrm{e}}^{2}$ e $\sigma_{\mathrm{a} 12}=$ componentes de variância aditivo direto e residual e de covariância aditiva direta, respectivamente.

$x 1.000$.

$\sigma_{a}^{2}, \sigma_{e}^{2} e \sigma_{a 12}=$ additive direct and residual components of variance, and additive direct covariance component, respectively.

daquelas de P12. Essas relações devem estar ligadas ao desempenho dos animais de acordo com a época de nascimento (Tabela 1). Verifica-se nessa tabela que a média e o desvio-padrão do peso à desmama são maiores para os animais nascidos no segundo semestre. Para o peso aos 12 meses de idade ocorre o contrário, ou seja, a média e o desvio-padrão são maiores para os animais nascidos no primeiro semestre. Para o GDA, que apresentou maior variância aditiva no semestre 2 , a maior média observada ocorre quando os animais nascem no semestre 1, mas os desvios-padrão são iguais.

Para as análises unicaráter (Tabela 2), as tendências das relações dos componentes de co(variâncias) entre épocas de nascimento são parecidas com aquelas das análises bicaráter, com exceção de CPG, que mostrou maior variação genética aditiva no semestre 2.

Os componentes de variância genéticos e residuais estimados pelas análises bicaráter foram numericamente diferentes daqueles obtidos pelas análises unicaráter, para todas as características estudadas (Tabela 2). As análises bicaráter apresentaram, proporcionalmente, valores maiores para a variância genética aditiva e valores menores para as variâncias residuais, implicando em maiores valores de herdabilidade (Tabela 3 ).

Estes resultados mostram que a análise bicaráter, pelo fato de utilizar matrizes de covariâncias fenotípicas das características, proporciona estimativas de (co)variâncias mais adequadas, confirmando as afirmações de Pollak (1985).

As estimativas de herdabilidade das características foram semelhantes nas duas épocas de nascimento, pelas análises bicaráter e apresentaram pequenas diferenças de magnitude, pelas análises unicaráter (Tabela 3). As correlações genéticas entre as duas épocas variaram de 0,87 a 0,97 , dependendo da característica, sendo, portanto, consideradas altas segundo os critérios de Robertson (1959), segundo o qual, quando a correlação genética para a mesma característica em ambientes diferentes é menor que 0,80 , existe interação genótipo x ambiente. Henderson (1984), entretanto, argumenta que em situações de correlação genética unitária ou próxima de um, com variâncias genéticas aditivas e residuais que variam sem proporcionalidade entre os ambientes, proporcionando estimativas de herdabilidade também variáveis de acordo com o ambiente, pequena interação genótipo x ambiente pode existir. Neste trabalho, houve diferenças nas variações das variâncias aditivas e residuais entre semestres, sugerindo a existência de interação.

Utilizando-se o índice $\mathrm{rr}=\mathrm{h}_{1} \mathrm{~h}_{2} \mathrm{r}_{\mathrm{g}} / \mathrm{h}_{1}{ }^{2}$ apresentado por Cameron (1997), que mede a resposta relativa na época 1 quando a seleção é baseada na época 2, e as estimativas de herdabilidade e de correlação genética obtidas pelas análises bicaráter (Tabela 3), obtêm-se valores iguais a 0,$86 ; 1,00 ; 1,06$ e 0,87 para $\mathrm{PD}, \mathrm{P} 12$, GDA e CPG, respectivamente. Se a seleção for baseada na época 1 , as respostas relativas na época 2 serão de 0,88 (PD), 0,95 (P12), 0,80 (GDA) e 0,87 (CPG). Estes valores indicam que selecionando-se com base no desempenho na época 1 a resposta vai ser menor para os animais criados na época 2. Somente para $\mathrm{P} 12$, a seleção com base na época 2

R. Bras. Zootec., v.34, n.2, p.489-495, 2005 
Tabela 3 - Estimativas de herdabilidades $\left(h_{a}{ }^{2}\right)$ e correlações genéticas $\left(\rho_{g}\right)$ para os pesos à desmama (PD) e aos 12 meses de idade (P12), ganho de peso médio diário da desmama aos 12 meses de idade (GDA) e primeiro componente principal (CPG) combinando PD, GDA e P12, para os animais Canchim nascidos no $1^{\circ}$ (S1) e no $2^{\circ}$ (S2) semestres do ano

Table 3 - Heritability $\left(h_{a}{ }^{2}\right)$ and genetic correlation $\left(\rho_{g}\right)$ estimates for weaning $(W W)$ and 12-month (W12) weights, daily gain from weaning to 12 months of age (ADG) and first principal component (PC) combining WW, W12 and ADG, for Canchim cattle born in the $1^{\text {st }}(S 1)$ and $2^{\text {nd }}($ S2) semesters

\begin{tabular}{|c|c|c|c|c|c|}
\hline \multirow{4}{*}{$\begin{array}{l}\text { Caract. } \\
\text { Trait }\end{array}$} & \multicolumn{3}{|c|}{$\begin{array}{l}\text { Análise bicaráter } \\
\text { Two-trait analysis }\end{array}$} & \multicolumn{2}{|c|}{$\begin{array}{l}\text { Análise unicaráter } \\
\text { One-trait analysis }\end{array}$} \\
\hline & S1 & $\mathrm{S} 2$ & S1 e S2 & S1 & $\mathrm{S} 2$ \\
\hline & $S 1$ & $S 2$ & $S 1$ and $S 2$ & $S 1$ & $S 2$ \\
\hline & $\mathrm{h}_{\mathrm{a}}{ }^{2}$ & $\mathrm{~h}_{\mathrm{a}}{ }^{2}$ & $\rho_{\mathrm{g}}$ & $\mathrm{h}_{\mathrm{a}}{ }^{2}$ & $\mathrm{~h}_{\mathrm{a}}{ }^{2}$ \\
\hline $\mathrm{PD}(W W)$ & 0,41 & 0,40 & 0,87 & 0,36 & 0,37 \\
\hline $\mathrm{P} 12(W 12)$ & 0,36 & 0,38 & 0,97 & 0,30 & 0,36 \\
\hline $\operatorname{GDA}(A D G)$ & 0,09 & 0,12 & 0,91 & 0,08 & 0,11 \\
\hline $\mathrm{CPG}(P C)$ & 0,44 & 0,44 & 0,88 & 0,37 & 0,41 \\
\hline
\end{tabular}

será equivalente à seleção com base na época 1, para promover mudanças genéticas nesta época. Estes resultados indicam, com exceção de P12, a existência de interação genótipo $\mathrm{x}$ ambiente.

Os coeficientes de correlação de Spearman entre os valores genéticos de touros que produziram progênie nos dois semestres do ano são apresentados na Tabela 4, para cada característica e tipo de análise (uni e bicaráter). Considerando-se as análises unicaráter, observa-se que esses coeficientes, apesar de significativos $(\mathrm{P}<0,0001)$, são de baixa magnitude, demonstrando que existe mudança na classificação dos animais de uma época (semestre) para a outra e que os valores genéticos dos animais nas duas épocas são bastante diferentes. Dos dez melhores touros classificados no semestre 1, apenas três, três, zero e dois estão entre os dez primeiro colocados no semestre 2, para PD, P12, GDA e CPG, respectivamente. Entretanto, considerando-se as análises bicaráter, as correlações de Spearman são altas, mostrando que os touros apresentam classificação semelhante nas duas épocas do ano. Neste caso, dos dez melhores touros classificados no semestre 1, apenas três, um, dois e quatro não estão entre os dez primeiros colocados no semestre 2, para PD, P12, GDA e CPG, respectivamente. Como houve mudanças nas classificações dos touros de um semestre para outro, associado ao fato de que as diferenças nos valores genéticos de um semestre para outro mudam de touro para touro, há indícios de interação genótipo $\mathrm{x}$ ambiente.

$\mathrm{Na}$ Tabela 5, são apresentados os componentes de variância, as herdabilidades e os valores das funções de verossimilhança, obtidos por meio do
Método 2 sem (Modelo 1) e com (Modelo 2) o efeito aleatório de touro - semestre de nascimento, para as características estudadas.

Verifica-se, pelo teste de $\chi^{2}$, que os dois modelos diferem para todas as características estudadas. A inclusão do efeito não correlacionado de touro época de nascimento (Modelo 2) aumentou o valor da função de verossimilhança, indicando que esse modelo é melhor do que o Modelo 1. Observa-se que os componentes de variância aditivo direto e residual foram menores para o Modelo 2. O efeito não correlacionado de touro - semestre de nascimento absorveu parte da variação genética aditiva direta, fazendo com que as estimativas de herdabilidade fossem menores para o Modelo 2, em comparação ao Modelo 1, o qual não incluiu o componente de touro - semestre de nascimento. Estes resultados indicam que o componente de variância de touro - semestre de nascimento $\left(\sigma_{\mathrm{te}}{ }^{2}\right)$ é importante para PD, P12, GDA e CPG, sugerindo a existência de interação genótipo $\mathrm{x}$ época de nascimento para essas características.

Comparando-se o Modelo 1 (análise unicaráter, Tabela 5) com o modelo completo da análise bicaráter do Método 1, por meio do teste de razão de verossimilhança, observam-se valores de $\chi^{2}$ iguais a 288, 439, 154 e 345 para PD, P12, GDA e CPG, respectivamente, todos significativos $(\mathrm{P}<0,01)$, indicando que o modelo bicaráter é mais apropriado do que o unicaráter, sugerindo a importância de se considerar as características nas duas épocas de nascimento como sendo características diferentes.

Os resultados obtidos neste trabalho concordam em parte com outros resultados sobre interação

R. Bras. Zootec., v.34, n.2, p.489-495, 2005 
Tabela 4 - Coeficientes de correlação de Spearman entre os valores genéticos de touros que possuem progênies nos dois semestres de nascimento, para os pesos à desmama (PD) e aos 12 meses de idade (P12), ganho de peso médio diário da desmama aos 12 meses de idade (GDA) e primeiro componente principal (CPG) combinando PD, GDA e P12, de acordo com o tipo de análise (uni e bicaráter)

Table 4 - Spearman rank correlation among the breeding values of the sires that had progeny in the two semesters, for weaning (WW) and 12-month (W12) weights, daily gain from weaning to 12 months of age (ADG) and first principal component (PC) combining WW, W12 and $A D G$, according to the analysis (one- or two-trait)

\begin{tabular}{lcccc}
\hline \multirow{5}{*}{ Análise } & \multicolumn{4}{c}{$\begin{array}{c}\text { Característica } \\
\text { Trait }\end{array}$} \\
\cline { 2 - 5 } Analysis & PD & P12 & GDA & CPG \\
\hline Unicaráter & 0,58 & W12 & $A D G$ & $P C$ \\
One-trait & $(222)$ & $(232)$ & $(232)$ & $(232)$ \\
Bicaráter & 0,97 & 1,00 & 0,99 & 0,97 \\
Two-trait & $(245)$ & $(247)$ & $(247)$ & $(247)$ \\
\hline
\end{tabular}

* Número de animais entre parênteses.

"Number of animals within parentheses. genótipo x ambiente reportados no Brasil. Alencar (1985), utilizando várias metodologias, não encontrou interação significativa entre touro e época de nascimento para os pesos ao nascimento e à desmama de bezerros da raça Canchim do mesmo rebanho deste estudo. Entretanto, houve mudança na classificação dos touros dentro de cada época de nascimento, quando eles foram ordenados por seus valores genéticos. Os coeficientes de herdabilidade estimados para cada época e todas as épocas em conjunto foram semelhantes, enquanto que as correlações genéticas variaram muito com a época de nascimento. Rosa et al. (1982) verificaram interação significativa touro $x$ sexo do bezerro para os pesos ao nascimento e aos 18 meses de idade de animais da raça Nelore. Nobre et al. (1987) estimaram herdabilidades e repetibilidades diferentes para diversas estações de nascimento, para o peso ao nascer de bezerros da raça Nelore. Nobre et al. (1988) encontraram interações touro x época de nascimento e touro $\mathrm{x}$ rebanho significativas, para os pesos até um ano de idade de bezerros da raça

Tabela 5 - Componentes de variância, herdabilidades e valor das funções de verossimilhança, obtidos por meio das análises sem (Modelo 1) e com (Modelo 2) o efeito aleatório de touro - semestre de nascimento, para os pesos à desmama (PD) e aos 12 meses de idade (P12), ganho de peso médio diário da desmama aos 12 meses de idade (GDA) e primeiro componente principal (CPG) combinando PD, GDA e P12

Table 5 - Components of variance, heritabilities, and likelyhood function values, obtained by the analyses without (Model 1) and with (Model 2) the sire - semester random effect, for weaning (WW) and 12-month (W12) weights, daily gain from weaning to 12 months of age (ADG) and first principal component (PC) combining WW, W12 and ADG

\begin{tabular}{|c|c|c|c|c|c|c|c|c|c|c|}
\hline Caract. & & odelc & Model & & & & Mod & $2(M$ & l2) & \\
\hline Trait & $\sigma_{a}^{2}$ & $\sigma_{\mathrm{e}}^{2}$ & $h^{2}$ & $-2 \log \mathrm{L}$ & $\sigma_{a}{ }^{2}$ & $\sigma_{t e^{2}}$ & $\sigma_{\mathrm{e}}^{2}$ & $h^{2}$ & $-2 \log \mathrm{L}$ & $\chi^{2}$ \\
\hline$\overline{\mathrm{PD}(W W)}$ & 327 & 573 & 0,36 & 41860 & 306 & 75 & 543 & 0,33 & 41765 & $95^{*}$ \\
\hline P12(W12) & 361 & 663 & 0,35 & 37867 & 336 & 71 & 639 & 0,32 & 37813 & $54 *$ \\
\hline $\mathrm{GDA}^{1}(A D G)$ & 5 & 30 & 0,13 & -10973 & 1 & 4 & 30 & 0,04 & -11076 & $103 *$ \\
\hline $\mathrm{CPG}(P C)$ & 496 & 801 & 0,38 & 38082 & 463 & 79 & 776 & 0,35 & 38036 & $46^{*}$ \\
\hline
\end{tabular}

$\sigma_{\mathrm{a}}{ }^{2}, \sigma_{\mathrm{e}}{ }^{2}, \sigma_{\mathrm{te}}{ }^{2} \mathrm{e} \mathrm{h}^{2}=$ componentes de variância aditivo direto, residual e de touro - época e herdabilidade, respectivamente.

$1 \times 1.000 .{ }^{*} \mathrm{P}<0,001$

$\sigma_{a}^{2}, \sigma_{e}^{2}, \sigma_{t e}{ }^{2} e h^{2}=$ additive direct, residual and sire - semester components of variance and heritability, respectively.

Nelore. Silva (1990) observou interação touro x região significativa para os pesos à desmama e aos 12 meses de idade e Euclides Filho et al. (1991) mostraram a importância da interação idade da vaca ao parto $\mathrm{x}$ reprodutor dentro de fazenda, para os pesos do nascimento aos 18 meses de idade. Souza et al. (2003) observaram interação significativa genótipo x região para o peso à desmama de bovinos da raça Nelore. Eler et al. (2000) verificaram efeito significa- tivo da interação touro $\mathrm{x}$ rebanho para os pesos ao nascimento e à desmama e para o ganho em peso da desmama ao sobreano na raça Nelore, havendo também efeito da interação sobre os componentes de variância e de covariância e sobre os parâmetros genéticos.

Neste trabalho, houve evidências de interação genótipo x época de nascimento para os pesos à desmama e aos 12 meses de idade, para o ganho em 
peso da desmama aos 12 meses de idade e para o índice, obtido de componentes principais, envolvendo essas três características. Entretanto, pelas análises bicaráter, observaram-se correlações genéticas altas para as características nos dois ambientes, respostas relativas altas em um ambiente quando a seleção é baseada no outro ambiente e pequenas mudanças na classificação dos touros nos dois ambientes. Dessa maneira, sugere-se que a interação genótipo x época de nascimento seja considerada por ocasião da avaliação genética de animais no rebanho estudado.

\section{Conclusões}

Existe interação genótipo x época (semestre) de nascimento dos animais do rebanho Canchim da Embrapa Pecuária Sudeste para PD, P12, GDA e CPG, indicando que a avaliação genética e a seleção de animais devem considerar essa interação.

\section{Literatura Citada}

ALENCAR, M.M. Estudo da interação touro x época de nascimento em um rebanho Canchim. Revista da Sociedade Brasileira de Zootecnia, v.14, n.2, p.224-234, 1985.

BOLDMAN, K.G.; KRIESE, L.A.; Van VLECK, L.D. et al.. A manual for use of MTDFREML. A set of programs to obtain estimates of variance and covariance. Lincoln: Department of Agriculture, Agricultural Research Service, 1993. 120p.

CAMERON, N.D. Selection indexes and prediction of genetics merit in animal breeding. Edinburgh: CAB International, 1997. 202p.

ELER, J.P.; FERRAZ, J.B.S.; GOLDEN, B.L. et al. Influência da interação touro $x$ rebanho na estimação da correlação entre efeitos genéticos direto e materno em bovinos da raça Nelore. Revista Brasileira de Zootecnia, v.29, n.6, p.16421648, 2000.

EUCLIDES FILHO, K.; NOBRE, P.R.C.; ROSA, A.N. Idade da vaca e suas inter-relações com a fazenda, reprodutor e sexo do bezerro. Revista da Sociedade Brasileira de Zootecnia, v.20, n.1, p.40-46, 1991.
HENDERSON, C. R. Applications of linear models in animal breeding. Ontário: University of Guelf, 1984. 462p.

MOOD, A.M.; GRAYBILL, F.A.; BOES, D.C. Tests of hypotheses. In: MOOD, A.M., GRAYBILL, F.A., BOES, D.C (Ed.). Introduction to the theory of statistics. Tokio: McGraw-Hill, 1974. p.401-470.

NOBRE, P.R.C.; EUCLIDES FILHO, K.; ROSA, A.N. Repetibilidade e herdabilidade do peso ao nascer do gado Nelore por estação de nascimento. Revista da Sociedade Brasileira de Zootecnia, v.16, n.4, p.352-363, 1987.

NOBRE, P.R.C.; ROSA, A.N.; EUCLIDES FILHO, K. Interações reprodutor $\mathrm{x}$ estação de nascimento e reprodutor $\mathrm{x}$ fazenda sobre características de crescimento de bezerros Nelore. Revista da Sociedade Brasileira de Zootecnia, Viçosa, MG, v.17, n.2, p.120-131, 1988.

POLLAK, E. J. 1985. Genetic evaluation of beef cattle from performance. In: SIMPÓSIO INTERNACIONAL DE PRODUÇÃO ANIMAL, 1., 1983, Ribeirão Preto. Anais... Ribeirão Preto: Sociedade Brasileira de Genética, 1983. p.73-82.

ROBERTSON, A. The sampling variance of the genetic correlation coefficient. Biometrics, v.15, n.3, p.469-485, 1959.

ROSA, A.N.; MARIANTE, A.S.; EUCLIDES FILHO, K. et al. Investigação sobre a interação: sexo da progênie x reprodutor, para algumas características quantitativas. In: REUNIÃO ANUAL DA SOCIEDADE BRASILEIRA DEZOOTECNIA, 19., 1982, Piracicaba. Anais... Piracicaba: Sociedade Brasileira de Zootecnia, 1982. p.221.

SILVA, L.O.C. Tendência genética e interação genótipo $\mathbf{x}$ ambiente em rebanhos Nelore, criados a pasto no Brasil/ Central. Viçosa, MG: Universidade Federal de Viçosa, 1990. 113p. Tese (Doutorado em Zootecnia) - Universidade Federal de Viçosa, 1990.

SOUZA, J.C.; GADINI, C.H., SILVA, L.O.C. et al. Estimates of genetic parameters and evaluation of genotype $\mathrm{x}$ environment interaction for weaning weight in Nellore cattle. Archivos Latinoamericanos de Produción Animal, v.11, n.2, p.94-100, 2003.

STATISTICAL ANALYSIS SYSTEM - SAS. Statistical analysis system: user's guide. Cary: 1996.

Recebido em: 03/09/03

Aceito em: 24/08/04 